\title{
Mathematical Modeling of Optimum 3 Step Stress Accelerated Life Testing for Generalized Pareto Distribution
}

\author{
Sadia Anwar*, Sana Shahab, Arif Ul Islam \\ Department of Statistics \& Operations Research, Aligarh Muslim University, Aligarh, U.P, India \\ Email address: \\ sadiaanwar97@yahoo.com (S. Anwar), sana.shahb@gmail.com (S. Shahab)
}

To cite this article:

Sadia Anwar, Sana Shahab, Arif U1 Islam. Mathematical Modeling of Optimum 3 Step Stress Accelerated Life Testing for Generalized Pareto Distribution. American Journal of Theoretical and Applied Statistics. Vol. 4, No. 3, 2015, pp. 163-169. doi: 10.11648/j.ajtas.20150403.22

\begin{abstract}
This article contains the optimum 3 step stress accelerated life test under cumulative exposure model. The lifetimes of test units are assumed to follow a generalized Pareto distribution. The scale parameter of the used failure time distribution at the constant stress level is assumed to have a log-linear and quadratic relationship with the stress. A comparison between linear plan and quadratic plan by maximum likelihood estimators for the different sample sizes is shown in the table. The optimum test plans is obtained by minimizing the asymptotic variance of the maximum likelihood estimator of the $100 P^{t h}$ percentile of the lifetime distribution at normal stress condition for the model parameters. Tables of optimum times of changing stress level for both plans are also obtained.
\end{abstract}

Keywords: Accelerated Life Testing, Generalized Pareto Distribution, Asymptotic Variance, Maximum Likelihood Estimate, Life Stress Relationship (Linear and Quadratic), Cumulative Exposure Model

\section{Introduction}

Due to the fast development in the technology, the manufacturing plan is continuously improving. For this reason it is difficult to obtain information about lifetime of products or materials with high reliability at the time of testing under normal conditions because testing under normal operating conditions require a very long period of time and need an extensive number of units under test. So it is generally very expensive and impractical to complete reliability testing under normal conditions. Hence, to handle these problems, the study of accelerated life test (ALT) has been developed. ALT makes it possible to quickly obtain information on the life distribution of products by inducing early failure with higher than normal stress. There are mainly three types of life test methods in accelerated life testing design. The first method is constant stress ALT, second is step-stress ALT and the third is Progressive stress ALT. In step stress accelerated life testing, the test items are subjected to successively higher levels of stress at pre-assigned test times. The level of stress is increased step by step until all items have failed or the test stops for other reasons.

There are usually two types of step stress accelerated life test (SSALT) plans, a simple SSALT and a multiple-step SSALT. In the simple SSALT there is a single change of stress during the test. Nelson (1990) presented the step stress scheme with cumulative exposure model. Miller and Nelson introduced (1983) a simple step-stress accelerated life test (SSALT) plan in an exponential cumulative exposure (CE) model. Bai et al. (1989) presented optimum simple stepstress accelerated life tests with censoring. Xiong (1999) has studied an exponential CE model with a threshold parameter in the simple SSALT. Lu et al. (2002) considered the Weibull CE model with the inverse power law in the simple SSALT. In the multiple-step SSALT changes of stress are more than once. Khamis and Higgins (1996) introduced first the optimum three-step SSALT plan using quadratic stress-life relationship assuming that the failure time follows an exponential distribution using. Khamis (1997) extended the 2-step stress work for m-step stress ALT with $\mathrm{k}$ stress variables using the exponential distribution, assuming complete knowledge of the stress-life relation with multiple stress variables. Khamis and Higgins (1998) proposed a new model for SSALT as an alternative to the CEM, which is based on a time transformation of the exponential CEM. McSorley et al. (2002) has introduced the performance of parameter-estimates in step stress accelerated life-tests with various sample-sizes. Fard and Chenhua (2009) studied the optimum step-stress accelerated life test design for reliability prediction using Khamis-Higgins $(\mathrm{K}-\mathrm{H})$ model. Hunt and $\mathrm{Xu}$ (2012) derived the optimum stress-changing time for the 
generalized K-H model by assuming that the lifetime of a test unit follows a Weibull distribution for type-I censored data. Alhadeed and Yang (2005) considered a simple SSALT plan for optimal time of changing stress level using the LogNormal distribution.

\section{The Model and Test Procedure}

\subsection{Generalized Pareto (GP) Distribution}

The generalized Pareto (GP) distribution is also known as the Pareto of second kind with two parameters or Lomax distribution. This distribution has been widely used in the field of reliability modeling and life testing. Bander AlZahrani (2012) presented the maximum likelihood estimation for generalized Pareto distribution under progressive censoring with binomial removals. The life times of the test items is said to have the generalized Pareto distribution if it has the probability density function (PDF)

$$
f(y)=\alpha \theta(1+\theta y)^{-(\alpha+1)} \quad y>0, \alpha, \theta>0
$$

where $\theta$ is the scale parameter and $\alpha$ is the shape parameter of the distribution.

Now, the cumulative distribution function is given by

$$
F(y)=1-(1+\theta y)^{-\alpha} \quad y>0, \alpha, \theta>0
$$

The survival function of the GP distribution is given by

$$
\bar{F}(y)=(1+\theta y)^{-\alpha} \quad y>0, \alpha, \theta>0
$$

And the corresponding hazard rate is given by

$$
h(y)=\frac{\alpha \theta}{(1+\theta y)}
$$

\subsection{SSALT with Cumulative Exposure Model}

According to the cumulative exposure model, the CDF in SSALT for 3-step is given by

$$
F(t)= \begin{cases}F_{1}(t) & 0 \leq \mathrm{t}<\tau_{1} \\ F_{2}\left(t-\tau_{1}+S_{1}\right) & \tau_{1} \leq t<\tau_{2} \\ F_{3}\left(t-\tau_{2}+S_{2}\right) & \tau_{2} \leq t<\infty\end{cases}
$$

with $S_{1}$ the solution of $F_{2}\left(S_{1}\right)=F_{1}\left(\tau_{1}\right)$ and $S_{2}$ the solution of $F_{3}\left(S_{2}\right)=F_{2}\left(\tau_{2}-\tau_{1}+S_{1}\right)$. On solving we get

$$
S_{1}=\frac{\theta_{1}}{\theta_{2}} \tau_{1} \text { and } S_{2}=\frac{\theta_{2}}{\theta_{3}}\left(\tau_{2}-\tau_{1}\right)+\frac{\theta_{1}}{\theta_{3}} \tau_{1}
$$

Hence Generalized cumulative exposure model for 3 step stress is as follow

$$
F(t)= \begin{cases}1-\left(1+\theta_{1} t\right)^{\alpha} & 0 \leq t<\tau_{1} \\ 1-\left[1+\theta_{2}\left(t-\tau_{1}\right)+\theta_{1} \tau_{1}\right]^{\alpha} & \tau_{1} \leq t<\tau_{2} \\ 1-\left[1+\theta_{3}\left(t-\tau_{2}\right)+\theta_{2}\left(\tau_{2}-\tau_{1}\right)+\theta_{1} \tau_{1}\right]^{\alpha} & \tau_{2} \leq t<\infty\end{cases}
$$

The corresponding PDF for the cumulative exposure model is given as above by equation (5)

$$
f(t)= \begin{cases}\alpha \theta_{1}\left(1+\theta_{1} t\right)^{-(\alpha+1)} & 0 \leq \mathrm{t}<\tau_{1} \\ \alpha \theta_{2}\left[1+\theta_{2}\left(t-\tau_{1}\right)+\theta_{1} \tau_{1}\right]^{-(\alpha+1)} & \tau_{1} \leq t<\tau_{2} \\ \alpha \theta_{3}\left[1+\theta_{3}\left(t-\tau_{2}\right)+\theta_{2}\left(\tau_{2}-\tau_{1}\right)+\theta_{1} \tau_{1}\right]^{-(\alpha+1)} & \tau_{2} \leq t<\infty\end{cases}
$$

\subsection{Assumptions}

1. Testing is performed at the three stress levels $S_{1}, S_{2}$ and $S_{3}$, where $S_{1}>S_{2}>S_{3}$.

2. The lifetime of the test units follow a generalized Pareto distribution under any stress.

3. The parameter $\alpha$ is independent of time and stress.

4. The scale parameter $\theta_{i}$ at stress level $i, i=1,2,3$ is a $\log$ linear function of stress given by (i) or (ii)

$$
\begin{aligned}
\log \theta_{i} & =a+b S_{i} \\
\log \theta_{i} & =a+b S_{i}+c S_{i}^{2}
\end{aligned}
$$

where, $a, b$ and $c$ are unknown parameters on the nature of the product and the test method.

A random sample of $\mathrm{n}$ identical units are initially placed on low stress $S_{1}$ and run until pre-specified time $\tau_{1}$ when the stress is changed to high stress $S_{2}$ for those remaining units that have not failed. The test is continued until pre-specified time $\tau_{2}$ when stress is changed to $S_{3}$, and continued until all remaining units fail

\section{Likelihood Function}

In order to obtain the MLE of the model parameters, let $t_{i j}$, $i=1,2,3, j=1,2,3, \ldots \ldots \ldots \ldots n_{i}$ be the observed failure time of a test unit $j$ under the stress level $i$, where $n_{i}$ denotes the number of units failed at the stress level $i$. The likelihood function is given by

$$
L\left(t, \alpha, \theta_{1}, \theta_{2}, \theta_{3}\right)=\prod_{j=1}^{n_{1}}\left[\alpha \theta_{1}\left(1+t_{1 j} \theta_{1}\right)^{-(\alpha+1)}\right] \prod_{j=1}^{n_{2}}\left[\alpha \theta_{2}\left\{1+\left(t_{2 j}-\tau_{1}\right) \theta_{2}+\tau_{1} \theta_{1}\right\}^{-(\alpha+1)}\right] \prod_{j=1}^{n_{3}}\left[\alpha \theta_{3}\left\{1+\left(t_{3 j}-\tau_{2}\right) \theta_{3}+\left(\tau_{2}-\tau_{1}\right) \theta_{2}+\tau_{1} \theta_{1}\right\}^{-(\alpha+1)}\right]
$$


Log likelihood function of equation (6) can be written as

$$
\begin{aligned}
& l=n \log \alpha+n_{1} \log \theta_{1}+n_{2} \log \theta_{2}+n_{3} \log \theta_{3} \\
& -(\alpha+1)\left\{\sum_{j=1}^{n_{1}} \log \left(1+t_{1 j} \theta_{1}\right)+\sum_{j=1}^{n_{2}} \log \left[1+\left(t_{2 j}-\tau_{1}\right) \theta_{2}+\tau_{1} \theta_{1}\right]\right. \\
& \left.+\sum_{j=1}^{n_{3}} \log \left[1+\left(t_{3 j}-\tau_{2}\right) \theta_{3}+\left(\tau_{2}-\tau_{1}\right) \theta_{2}+\tau_{1} \theta_{1}\right]\right\} \\
& l=n \log \alpha+n_{1}\left(a+b S_{1}\right)+n_{2}\left(a+b S_{2}\right)+n_{3}\left(a+b S_{3}\right) \\
& -(\alpha+1)\left\{\sum_{j=1}^{n_{1}} \log \left(1+t_{1 j} e^{a+b S_{1}}\right)+\sum_{j=1}^{n_{2}} \log \left[1+\left(t_{2 j}-\tau_{1}\right) e^{a+b S_{2}}+\tau_{1} e^{a+b S_{1}}\right]\right. \\
& \left.+\sum_{j=1}^{n_{3}} \log \left[1+\left(t_{3 j}-\tau_{2}\right) e^{a+b S_{3}}+\left(\tau_{2}-\tau_{1}\right) e^{a+b S_{2}}+\tau_{1} e^{a+b S_{1}}\right]\right\}
\end{aligned}
$$

\section{Optimum Linear Step-Stress Test}

Using the relation $\log \theta_{i}=a+b S_{i}, i=1,2,3$, the $\log$

MLEs of $a$ and $b$ are obtained by solving the equations $\frac{\partial l}{\partial a}=0$ and $\frac{\partial l}{\partial b}=0$.

$$
\begin{aligned}
& \frac{\partial l}{\partial a}=n-(\alpha+1)\left\{\sum_{j=1}^{n_{1}} \frac{t_{1 j} \theta_{1}}{\left(1+t_{1 j} \theta_{1}\right)}+\sum_{j=1}^{n_{2}} \frac{\left(t_{2 j}-\tau_{1}\right) \theta_{2}+\tau_{1} \theta_{1}}{\left[1+\left(t_{2 j}-\tau_{1}\right) \theta_{2}+\tau_{1} \theta_{1}\right]}+\sum_{j=1}^{n_{3}} \frac{\left(t_{3 j}-\tau_{2}\right) \theta_{3}+\left(\tau_{2}-\tau_{1}\right) \theta_{2}+\tau_{1} \theta_{1}}{\left[1+\left(t_{3 j}-\tau_{2}\right) \theta_{3}+\left(\tau_{2}-\tau_{1}\right) \theta_{2}+\tau_{1} \theta_{1}\right]}\right\} \\
& \frac{\partial l}{\partial b}=n_{1} S_{1}+n_{2} S_{2}+n_{3} S_{3} \\
& -(\alpha+1)\left\{\sum_{j=1}^{n_{1}} \frac{S_{1} t_{1 j} \theta_{1}}{\left(1+t_{1 j} \theta_{1}\right)}+\sum_{j=1}^{n_{2}} \frac{S_{2}\left(t_{2 j}-\tau_{1}\right) \theta_{2}+S_{1} \tau_{1} \theta_{1}}{\left[1+\left(t_{2 j}-\tau_{1}\right) \theta_{2}+\tau_{1} \theta_{1}\right]}+\sum_{j=1}^{n_{3}} \frac{S_{3}\left(t_{3 j}-\tau_{2}\right) \theta_{3}+S_{2}\left(\tau_{2}-\tau_{1}\right) \theta_{2}+S_{1} \tau_{1} \theta_{1}}{\left[1+\left(t_{3 j}-\tau_{2}\right) \theta_{3}+\left(\tau_{2}-\tau_{1}\right) \theta_{2}+\tau_{1} \theta_{1}\right]}\right\} \\
& \frac{\partial^{2} l}{\partial a^{2}}=-(\alpha+1)\left\{\sum_{j=1}^{n_{1}} \frac{t_{1 j} \theta_{1}}{\left(1+t_{1 j} \theta_{1}\right)^{2}}+\sum_{j=1}^{n_{2}} \frac{\left(t_{2 j}-\tau_{1}\right) \theta_{2}+\tau_{1} \theta_{1}}{\left[1+\left(t_{2 j}-\tau_{1}\right) \theta_{2}+\tau_{1} \theta_{1}\right]^{2}}+\sum_{j=1}^{n_{3}} \frac{\left(t_{3 j}-\tau_{2}\right) \theta_{3}+\left(\tau_{2}-\tau_{1}\right) \theta_{2}+\tau_{1} \theta_{1}}{\left[1+\left(t_{3 j}-\tau_{2}\right) \theta_{3}+\left(\tau_{2}-\tau_{1}\right) \theta_{2}+\tau_{1} \theta_{1}\right]^{2}}\right\} \\
& \frac{\partial^{2} l}{\partial b^{2}}=-(\alpha+1)\left\{\sum_{j=1}^{n_{1}} \frac{S_{1}^{2} t_{1 j} \theta_{1}}{\left(1+t_{1 j} \theta_{1}\right)^{2}}+\sum_{j=1}^{n_{2}} \frac{S_{2}^{2}\left(t_{2 j}-\tau_{1}\right) \theta_{2}+S_{1}^{2} \tau_{1} \theta_{1}+\left(S_{1}^{2}+S_{2}^{2}-2 S_{1} S_{2}\right) \theta_{1} \theta_{2} \tau_{1}\left(t_{2 j}-\tau_{1}\right)}{\left[1+\left(t_{2 j}-\tau_{1}\right) \theta_{2}+\tau_{1} \theta_{1}\right]^{2}}\right. \\
& \left.+\sum_{j=1}^{n_{3}} \frac{\left[\begin{array}{l}
S_{3}^{2}\left(t_{3 j}-\tau_{2}\right) \theta_{3}+S_{2}^{2}\left(\tau_{2}-\tau_{1}\right) \theta_{2}+S_{1}^{2} \tau_{1} \theta_{1}+\left(S_{1}^{2}+S_{3}^{2}-2 S_{1} S_{3}\right) \theta_{1} \theta_{3} \tau_{1}\left(t_{3 j}-\tau_{2}\right) \\
+\left(S_{1}^{2}+S_{2}^{2}-2 S_{1} S_{2}\right) \theta_{1} \theta_{2} \tau_{1}\left(\tau_{2}-\tau_{1}\right)+\left(S_{2}^{2}+S_{3}^{2}-2 S_{2} S_{3}\right) \theta_{2} \theta_{3}\left(t_{3 j}-\tau_{2}\right)\left(\tau_{2}-\tau_{1}\right)
\end{array}\right]}{\left[1+\left(t_{3 j}-\tau_{2}\right) \theta_{3}+\left(\tau_{2}-\tau_{1}\right) \theta_{2}+\tau_{1} \theta_{1}\right]^{2}}\right\} \\
& \frac{\partial^{2} l}{\partial a \partial b}=-(\alpha+1)\left\{\sum_{j=1}^{n_{1}} \frac{S_{1} t_{1 j} \theta_{1}}{\left(1+t_{1 j} \theta_{1}\right)^{2}}+\sum_{j=1}^{n_{2}} \frac{S_{2}\left(t_{2 j}-\tau_{1}\right) \theta_{2}+S_{1} \tau_{1} \theta_{1}}{\left[1+\left(t_{2 j}-\tau_{1}\right) \theta_{2}+\tau_{1} \theta_{1}\right]^{2}}+\sum_{j=1}^{n_{3}} \frac{S_{3}\left(t_{3 j}-\tau_{2}\right) \theta_{3}+S_{2}\left(\tau_{2}-\tau_{1}\right) \theta_{2}+S_{1} \tau_{1} \theta_{1}}{\left[1+\left(t_{3 j}-\tau_{2}\right) \theta_{3}+\left(\tau_{2}-\tau_{1}\right) \theta_{2}+\tau_{1} \theta_{1}\right]^{2}}\right\}
\end{aligned}
$$

The Fisher information matrix is obtained by taking the negative second partial derivatives of the log-likelihood 


$$
F_{1}=\left[\begin{array}{cc}
-\frac{\partial^{2} l}{\partial a^{2}} & -\frac{\partial^{2} l}{\partial a \partial b} \\
-\frac{\partial^{2} l}{\partial b \partial a} & -\frac{\partial^{2} l}{\partial b^{2}}
\end{array}\right]
$$

The $\log$ of the $100 p^{\text {th }}$ percentile of the lifetime $t_{p}\left(S_{0}\right)$ at the design stress $S_{0}$ is given by

$$
\hat{\xi}\left(S_{0}\right)=\log \left(t_{p}\left(S_{0}\right)\right)=a+b S_{0}+\log \left(\frac{(1-P)^{-\frac{1}{\alpha}}-1}{\theta_{i}}\right)
$$

The Asymptotic variance is given by

$$
\begin{gathered}
A V_{1}\left(\hat{\xi}\left(S_{0}\right)\right)=A V_{1}\left(\log \left(t_{p}\left(S_{0}\right)\right)\right) \quad \begin{array}{l}
\text { For this quadratic model, the testing c } \\
\text { step, step-stress ALT. Thus, the log lik } \\
\text { can be extended to the quadratic model } \\
\text { value of } \theta_{i} \text { from equation (14) given by }
\end{array} \\
\left.=A V_{1}\left(\hat{a}+\hat{b} S_{0}+\log \left(\frac{(1-P)^{-\frac{1}{\alpha}}-1}{\hat{\theta}_{i}}\right)\right)=H F_{1}^{-1} H^{\prime} \quad(13)\right) \\
l=n \log \alpha+n_{1}\left(a+b S_{1}+c S_{1}^{2}\right)+n_{2}\left(a+b S_{2}+c S_{2}^{2}\right) \\
+n_{3}\left(a+b S_{3}+c S_{3}^{2}\right)-(\alpha+1)\left\{\sum_{j=1}^{n_{1}} \log \left[1+t_{1 j} e^{a+b S_{1}+c S_{1}^{2}}\right]+\right. \\
\sum_{\mathrm{j}=1}^{n_{2}} \log \left[1+\left(t_{2 j}-\tau_{1}\right) e^{a+b S_{2}+c S_{2}^{2}}+\tau_{1} e^{a+b S_{1}+c S_{1}^{2}}\right]+ \\
\left.\sum_{\mathrm{j}=1}^{n_{3}} \log \left[1+\left(t_{3 j}-\tau_{2}\right) e^{a+b S_{3}+c S_{3}^{2}}+\left(\tau_{2}-\tau_{1}\right) e^{a+b S_{2}+c S_{2}^{2}}+\tau_{1} e^{a+b S_{1}+c S_{1}^{2}}\right]\right\}
\end{gathered}
$$

Where $H=\left[\begin{array}{lll}\frac{\partial \hat{\xi}\left(S_{0}\right)}{\partial \hat{a}} & \frac{\partial \hat{\xi}\left(S_{0}\right)}{\partial \hat{b}} & \frac{\partial \hat{\xi}\left(S_{0}\right)}{\partial \hat{\alpha}}\end{array}\right]$ and $F_{1}$ is the asymptotic fisher information matrix.

Therefore, the optimal times $\tau_{1}$ and $\tau_{2}$ to change stress levels under different values ofstresses and sample sizes will be obtained numerically using equation (13) which minimizes $A V_{1}\left(\hat{\xi}\left(S_{0}\right)\right)$.

\section{Optimum Quadratic Step-Stress Test}

For the quadratic model, using the log linear relationship

$$
\log \theta_{i}=a+b S_{i}+c S_{i}^{2}, i=1,2,3 .
$$

For this quadratic model, the testing can be done using a 3step, step-stress ALT. Thus, the log likelihood function (7) can be extended to the quadratic model after substituting the

MLEs of $a, b$ and $c$ are obtained by solving the equations $\frac{\partial l}{\partial a}=0, \frac{\partial l}{\partial b}=0$ and $\frac{\partial l}{\partial c}=0$.

$$
\begin{aligned}
\frac{\partial l}{\partial a}= & n-(\alpha+1)\left\{\sum_{j=1}^{n_{1}} \frac{t_{1 j} \theta_{1}}{\left(1+t_{1 j} \theta_{1}\right)}+\sum_{j=1}^{n_{2}} \frac{\left(t_{2 j}-\tau_{1}\right) \theta_{2}+\tau_{1} \theta_{1}}{\left[1+\left(t_{2 j}-\tau_{1}\right) \theta_{2}+\tau_{1} \theta_{1}\right]}+\sum_{j=1}^{n_{3}} \frac{\left(t_{3 j}-\tau_{2}\right) \theta_{3}+\left(\tau_{2}-\tau_{1}\right) \theta_{2}+\tau_{1} \theta_{1}}{\left[1+\left(t_{3 j}-\tau_{2}\right) \theta_{3}+\left(\tau_{2}-\tau_{1}\right) \theta_{2}+\tau_{1} \theta_{1}\right]}\right\} \\
\frac{\partial l}{\partial b}= & n_{1} S_{1}+n_{2} S_{2}+n_{3} S_{3} \\
& -(\alpha+1)\left\{\sum_{j=1}^{n_{1}} \frac{S_{1} t_{1 j} \theta_{1}}{\left(1+t_{1 j} \theta_{1}\right)}+\sum_{j=1}^{n_{2}} \frac{S_{2}\left(t_{2 j}-\tau_{1}\right) \theta_{2}+S_{1} \tau_{1} \theta_{1}}{\left[1+\left(t_{2 j}-\tau_{1}\right) \theta_{2}+\tau_{1} \theta_{1}\right]}+\sum_{j=1}^{n_{3}} \frac{S_{3}\left(t_{3 j}-\tau_{2}\right) \theta_{3}+S_{2}\left(\tau_{2}-\tau_{1}\right) \theta_{2}+S_{1} \tau_{1} \theta_{1}}{\left[1+\left(t_{3 j}-\tau_{2}\right) \theta_{3}+\left(\tau_{2}-\tau_{1}\right) \theta_{2}+\tau_{1} \theta_{1}\right]}\right\} \\
\frac{\partial l}{\partial c}= & n_{1} S_{1}^{2}+n_{2} S_{2}^{2}+n_{3} S_{3}^{3} \\
& -(\alpha+1)\left\{\sum_{j=1}^{n_{1}} \frac{S_{1}^{2} t_{1 j} \theta_{1}}{\left(1+t_{1 j} \theta_{1}\right)}+\sum_{j=1}^{n_{2}} \frac{S_{2}^{2}\left(t_{2 j}-\tau_{1}\right) \theta_{2}+S_{1}^{2} \tau_{1} \theta_{1}}{\left[1+\left(t_{2 j}-\tau_{1}\right) \theta_{2}+\tau_{1} \theta_{1}\right]}+\sum_{j=1}^{n_{3}} \frac{S_{3}^{2}\left(t_{3 j}-\tau_{2}\right) \theta_{3}+S_{2}^{2}\left(\tau_{2}-\tau_{1}\right) \theta_{2}+S_{1}^{2} \tau_{1} \theta_{1}}{\left[1+\left(t_{3 j}-\tau_{2}\right) \theta_{3}+\left(\tau_{2}-\tau_{1}\right) \theta_{2}+\tau_{1} \theta_{1}\right]}\right\}
\end{aligned}
$$




$$
\begin{aligned}
& \frac{\partial^{2} l}{\partial a^{2}}=-(\alpha+1)\left\{\sum_{j=1}^{n_{1}} \frac{t_{1 j} \theta_{1}}{\left(1+t_{1 j} \theta_{1}\right)^{2}}+\sum_{j=1}^{n_{2}} \frac{\left(t_{2 j}-\tau_{1}\right) \theta_{2}+\tau_{1} \theta_{1}}{\left[1+\left(t_{2 j}-\tau_{1}\right) \theta_{2}+\tau_{1} \theta_{1}\right]^{2}}+\sum_{j=1}^{n_{3}} \frac{\left(t_{3 j}-\tau_{2}\right) \theta_{3}+\left(\tau_{2}-\tau_{1}\right) \theta_{2}+\tau_{1} \theta_{1}}{\left[1+\left(t_{3 j}-\tau_{2}\right) \theta_{3}+\left(\tau_{2}-\tau_{1}\right) \theta_{2}+\tau_{1} \theta_{1}\right]^{2}}\right\} \\
& \frac{\partial^{2} l}{\partial b^{2}}=-(\alpha+1)\left\{\sum_{j=1}^{n_{1}} \frac{S_{1}^{2} t_{1 j} \theta_{1}}{\left(1+t_{1 j} \theta_{1}\right)^{2}}+\sum_{j=1}^{n_{2}} \frac{S_{2}^{2}\left(t_{2 j}-\tau_{1}\right) \theta_{2}+S_{1}^{2} \tau_{1} \theta_{1}+\left(S_{1}^{2}+S_{2}^{2}-2 S_{1} S_{2}\right) \theta_{1} \theta_{2} \tau_{1}\left(t_{2 j}-\tau_{1}\right)}{\left[1+\left(t_{2 j}-\tau_{1}\right) \theta_{2}+\tau_{1} \theta_{1}\right]^{2}}\right. \\
& \left.+\sum_{j=1}^{n_{3}} \frac{\left[\begin{array}{l}
S_{3}^{2}\left(t_{3 j}-\tau_{2}\right) \theta_{3}+S_{2}^{2}\left(\tau_{2}-\tau_{1}\right) \theta_{2}+S_{1}^{2} \tau_{1} \theta_{1}+\left(S_{1}^{2}+S_{3}^{2}-2 S_{1} S_{3}\right) \theta_{1} \theta_{3} \tau_{1}\left(t_{3 j}-\tau_{2}\right) \\
+\left(S_{1}^{2}+S_{2}^{2}-2 S_{1} S_{2}\right) \theta_{1} \theta_{2} \tau_{1}\left(\tau_{2}-\tau_{1}\right)+\left(S_{2}^{2}+S_{3}^{2}-2 S_{2} S_{3}\right) \theta_{2} \theta_{3}\left(t_{3 j}-\tau_{2}\right)\left(\tau_{2}-\tau_{1}\right)
\end{array}\right]}{\left[1+\left(t_{3 j}-\tau_{2}\right) \theta_{3}+\left(\tau_{2}-\tau_{1}\right) \theta_{2}+\tau_{1} \theta_{1}\right]^{2}}\right\} \\
& \frac{\partial^{2} l}{\partial c^{2}}=-(\alpha+1)\left\{\sum_{j=1}^{n_{1}} \frac{S_{1}^{4} t_{1 j} \theta_{1}}{\left(1+t_{1 j} \theta_{1}\right)^{2}}+\sum_{j=1}^{n_{2}} \frac{S_{2}^{4}\left(t_{2 j}-\tau_{1}\right) \theta_{2}+S_{1}^{4} \tau_{1} \theta_{1}+\left(S_{1}^{4}+S_{2}^{4}-2 S_{1}^{2} S_{2}^{2}\right) \theta_{1} \theta_{2} \tau_{1}\left(t_{2 j}-\tau_{1}\right)}{\left[1+\left(t_{2 j}-\tau_{1}\right) \theta_{2}+\tau_{1} \theta_{1}\right]^{2}}\right. \\
& \left.+\sum_{j=1}^{n_{3}} \frac{\left.\begin{array}{l}
S_{3}^{4}\left(t_{3 j}-\tau_{2}\right) \theta_{3}+S_{2}^{4}\left(\tau_{2}-\tau_{1}\right) \theta_{2}+S_{1}^{4} \tau_{1} \theta_{1}+\left(S_{1}^{4}+S_{3}^{4}-2 S_{1}^{2} S_{3}^{2}\right) \theta_{1} \theta_{3} \tau_{1}\left(t_{3 j}-\tau_{2}\right) \\
+\left(S_{1}^{4}+S_{2}^{4}-2 S_{1}^{2} S_{2}^{2}\right) \theta_{1} \theta_{2} \tau_{1}\left(\tau_{2}-\tau_{1}\right)+\left(S_{2}^{4}+S_{3}^{4}-2 S_{2}^{2} S_{3}^{2}\right) \theta_{2} \theta_{3}\left(t_{3 j}-\tau_{2}\right)\left(\tau_{2}-\tau_{1}\right)
\end{array}\right]}{\left[1+\left(t_{3 j}-\tau_{2}\right) \theta_{3}+\left(\tau_{2}-\tau_{1}\right) \theta_{2}+\tau_{1} \theta_{1}\right]^{2}}\right\} \\
& \frac{\partial^{2} l}{\partial a \partial b}=-(\alpha+1)\left\{\sum_{j=1}^{n_{1}} \frac{S_{1} t_{1 j} \theta_{1}}{\left(1+t_{1 j} \theta_{1}\right)^{2}}+\sum_{j=1}^{n_{2}} \frac{S_{2}\left(t_{2 j}-\tau_{1}\right) \theta_{2}+S_{1} \tau_{1} \theta_{1}}{\left[1+\left(t_{2 j}-\tau_{1}\right) \theta_{2}+\tau_{1} \theta_{1}\right]^{2}}+\sum_{j=1}^{n_{3}} \frac{S_{3}\left(t_{3 j}-\tau_{2}\right) \theta_{3}+S_{2}\left(\tau_{2}-\tau_{1}\right) \theta_{2}+S_{1} \tau_{1} \theta_{1}}{\left[1+\left(t_{3 j}-\tau_{2}\right) \theta_{3}+\left(\tau_{2}-\tau_{1}\right) \theta_{2}+\tau_{1} \theta_{1}\right]^{2}}\right\} \\
& \frac{\partial^{2} l}{\partial \alpha \partial c}=-(\alpha+1)\left\{\sum_{j=1}^{n_{1}} \frac{S_{1}^{2} t_{1 j} \theta_{1}}{\left(1+t_{1 j} \theta_{1}\right)^{2}}+\sum_{j=1}^{n_{2}} \frac{S_{2}^{2}\left(t_{2 j}-\tau_{1}\right) \theta_{2}+S_{1}^{2} \tau_{1} \theta_{1}}{\left[1+\left(t_{2 j}-\tau_{1}\right) \theta_{2}+\tau_{1} \theta_{1}\right]^{2}}+\sum_{j=1}^{n_{3}} \frac{S_{3}^{2}\left(t_{3 j}-\tau_{2}\right) \theta_{3}+S_{2}^{2}\left(\tau_{2}-\tau_{1}\right) \theta_{2}+S_{1}^{2} \tau_{1} \theta_{1}}{\left[1+\left(t_{3 j}-\tau_{2}\right) \theta_{3}+\left(\tau_{2}-\tau_{1}\right) \theta_{2}+\tau_{1} \theta_{1}\right]^{2}}\right\} \\
& \frac{\partial^{2} l}{\partial b \partial c}=-(\alpha+1)\left\{\sum_{j=1}^{n_{1}} \frac{S_{1}^{3} t_{1 j} \theta_{1}}{\left(1+t_{1 j} \theta_{1}\right)^{2}}+\sum_{j=1}^{n_{2}} \frac{S_{2}^{3}\left(t_{2 j}-\tau_{1}\right) \theta_{2}+S_{1}^{3} \tau_{1} \theta_{1}+\left(S_{1}^{3}+S_{2}^{3}-S_{1}^{2} S_{2}-S_{1} S_{2}^{2}\right) \theta_{1} \theta_{2} \tau_{1}\left(t_{2 j}-\tau_{1}\right)}{\left[1+\left(t_{2 j}-\tau_{1}\right) \theta_{2}+\tau_{1} \theta_{1}\right]^{2}}\right. \\
& +\sum_{j=1}^{n_{3}} \frac{\left[\begin{array}{l}
S_{3}^{3}\left(t_{3 j}-\tau_{2}\right) \theta_{3}+S_{2}^{3}\left(\tau_{2}-\tau_{1}\right) \theta_{2}+S_{1}^{3} \tau_{1} \theta_{1}+\left(S_{1}^{3}+S_{3}^{3}-S_{1}^{2} S_{3}-S_{1} S_{3}^{2}\right) \theta_{1} \theta_{3} \tau_{1}\left(t_{3 j}-\tau_{2}\right) \\
+\left(S_{1}^{3}+S_{2}^{3}-S_{1}^{2} S_{2}-S_{1} S_{2}^{2}\right) \theta_{1} \theta_{2} \tau_{1}\left(\tau_{2}-\tau_{1}\right)+\left(S_{2}^{3}+S_{3}^{3}-S_{2}^{2} S_{3}-S_{2} S_{3}^{2}\right) \theta_{2} \theta_{3}\left(t_{3 j}-\tau_{2}\right)\left(\tau_{2}-\tau_{1}\right)
\end{array}\right]}{\left[1+\left(t_{3 j}-\tau_{2}\right) \theta_{3}+\left(\tau_{2}-\tau_{1}\right) \theta_{2}+\tau_{1} \theta_{1}\right]^{2}}
\end{aligned}
$$

Fisher Information matrix is given by

$$
F_{2}=\left[\begin{array}{ccc}
-\frac{\partial^{2} l}{\partial a^{2}} & -\frac{\partial^{2} l}{\partial a \partial b} & -\frac{\partial^{2} l}{\partial a \partial c} \\
-\frac{\partial^{2} l}{\partial b \partial a} & -\frac{\partial^{2} l}{\partial b^{2}} & -\frac{\partial^{2} l}{\partial b \partial c} \\
-\frac{\partial^{2} l}{\partial c \partial a} & -\frac{\partial^{2} l}{\partial c \partial b} & -\frac{\partial l}{\partial c^{2}}
\end{array}\right]
$$

Here the Optimum criterion is to obtain optimum stress change time $\tau_{1}$ and $\tau_{2}$. The $\log$ of the $100 \mathrm{P}^{\text {th }}$ percentile of the lifetime $t_{p}\left(S_{0}\right)$ at the design stress $S_{0}$ is given as

$$
\hat{\xi}\left(S_{0}\right)=\log \left(t_{p}\left(S_{0}\right)\right)=a+b S_{0}+c S_{0}^{2}+\log \left(\frac{(1-P)^{-\frac{1}{\alpha}}-1}{\theta_{i}}\right)
$$

The asymptotic variance at the design stress $\mathrm{S}_{0}$ is then given by 


$$
A V_{2}\left(\hat{\xi}\left(S_{0}\right)\right)=H F_{2}^{-1} H^{\prime}
$$

Where $H=\left[\begin{array}{llll}\frac{\partial \hat{\xi}\left(S_{0}\right)}{\partial \hat{a}} & \frac{\partial \hat{\xi}\left(S_{0}\right)}{\partial \hat{b}} & \frac{\partial \hat{\xi}\left(S_{0}\right)}{\partial \hat{c}} & \frac{\partial \hat{\xi}\left(S_{0}\right)}{\partial \hat{\alpha}}\end{array}\right]$ and $F_{2}$ is the fisher information matrix

Therefore, the optimal times $\tau_{1}$ and $\tau_{2}$ will be obtained numerically using equation (25) which minimizes $A V_{2}\left(\hat{\xi}\left(S_{0}\right)\right)$.

Table 1. The Maximum likelihood estimate and Mean Absolute Error for $\alpha=0.0087$.

\begin{tabular}{|c|c|c|c|c|c|}
\hline \multirow{2}{*}{\multicolumn{2}{|c|}{ n Parameter }} & \multicolumn{2}{|c|}{ Quadratic case } & \multicolumn{2}{|c|}{ Linear case } \\
\hline & & Estimate & MAE & Estimate & MAE \\
\hline \multirow{3}{*}{20} & $\hat{\theta}_{1}$ & 4.67251 & 0.0089 & 5.98271 & 0.0998 \\
\hline & $\hat{\theta}_{2}$ & 4.73645 & 0.0731 & 5.39875 & 0.8237 \\
\hline & $\hat{\theta}_{3}$ & 2.73511 & 0.0874 & - & - \\
\hline \multirow{3}{*}{60} & $\hat{\theta}_{1}$ & 4.19484 & 0.0046 & 5.76513 & 0.0957 \\
\hline & $\hat{\theta}_{2}$ & 5.08365 & 0.0347 & 5.28436 & 0.6893 \\
\hline & $\hat{\theta}_{3}$ & 2.47452 & 0.0665 & - & - \\
\hline \multirow{3}{*}{80} & $\hat{\theta}_{1}$ & 4.08635 & 0.0038 & 5.57826 & 0.0765 \\
\hline & $\hat{\theta}_{2}$ & 4.71847 & 0.0043 & 5.26411 & 0.4796 \\
\hline & $\hat{\theta}_{3}$ & 2.27371 & 0.0569 & - & - \\
\hline \multirow{3}{*}{100} & $\hat{\theta}_{1}$ & 3.56356 & 0.0031 & 5.53912 & 0.0709 \\
\hline & $\hat{\theta}_{2}$ & 4.37163 & 0.0067 & 5.24716 & 0.2987 \\
\hline & $\hat{\theta}_{3}$ & 2.23144 & 0.0553 & - & - \\
\hline \multirow{3}{*}{120} & $\hat{\theta}_{1}$ & 3.25262 & 0.0008 & 5.51931 & 0.0035 \\
\hline & $\hat{\theta}_{2}$ & 4.08947 & 0.0021 & 5.18754 & 0.1098 \\
\hline & $\hat{\theta}_{3}$ & 2.20937 & 0.0289 & - & - \\
\hline \multirow{3}{*}{200} & $\hat{\theta}_{1}$ & 2.86746 & $1.298 \mathrm{e}^{-4}$ & 5.38756 & 0.0028 \\
\hline & $\hat{\theta}_{2}$ & 3.74562 & 0.0010 & 4.76581 & 0.1052 \\
\hline & $\hat{\theta}_{3}$ & 1.58456 & 0.0112 & - & - \\
\hline
\end{tabular}

\section{Simulation Study}

A numerical study was conducted in order to investigate the existence of the optimal stress change points and to evaluate them as a function of varying parameters. Simulations are performed to investigate the performances of the MLEs through their mean absolute error (MAE) for both relationships. Comparison between both plans is shown by calculating efficiencies. Table 1 present the Maximum likelihood estimates for $\mathrm{n}=20,60,80,100$ and 120 and their respective Mean absolute Error for both Quadratic and Linear relationship. Table 2 presents the results of optimal design of step-stress ALT for different sized samples and finally Table 3 Compound Linear Test-Plan Efficiencies.
Table 2. The results of optimal design of step-stress ALT for different sized sample.

\begin{tabular}{lllllll}
\hline \multirow{n}{n}{} & \multicolumn{3}{c}{$\mathbf{a}=\mathbf{0 . 6 9} \mathbf{b}=\mathbf{1 . 2 5}$} & \multicolumn{5}{c}{$\mathbf{a = 0 . 6 9}$} & $\mathbf{b}=\mathbf{1 . 2 5} \mathbf{c = 2 . 8 2}$ \\
\cline { 2 - 7 } & $\mathbf{n A V _ { 1 }}$ & $\boldsymbol{\tau}_{\mathbf{1}}$ & $\boldsymbol{\tau}_{\mathbf{2}}$ & $\mathbf{n} \mathbf{A V _ { 2 }}$ & $\boldsymbol{\tau}_{\mathbf{1}}$ & $\boldsymbol{\tau}_{\mathbf{2}}$ \\
\hline 20 & 0.232 & 3.31 & 3.99 & 0.192 & 2.38 & 3.00 \\
60 & 0.209 & 3.10 & 3.99 & 0.190 & 2.09 & 2.86 \\
80 & 0.187 & 2.88 & 3.01 & 0.089 & 1.83 & 1.83 \\
100 & 0.183 & 2.63 & 2.78 & 0.083 & 1.74 & 1.83 \\
120 & 0.077 & 2.25 & 2.61 & 0.003 & 1.74 & 1.83 \\
200 & 0.026 & 2.22 & 2.61 & $1.823 \mathrm{e}^{-3}$ & 1.74 & 1.83 \\
\hline
\end{tabular}

Table 3. Compound Linear Test Plan Efficiencies

\begin{tabular}{|c|c|c|c|c|c|}
\hline & & & & Efficiencies & \\
\hline$S_{1}$ & $\mathbf{S}_{\mathbf{2}}$ & $\mathbf{S}_{3}$ & $\mathbf{n}$ & Opt Linear & Opt Quadratic \\
\hline \multirow{6}{*}{2.58} & \multirow{6}{*}{3.58} & \multirow{6}{*}{4.58} & 20 & 0.67 & 0.74 \\
\hline & & & 60 & 0.62 & 0.77 \\
\hline & & & 80 & 0.79 & 0.79 \\
\hline & & & 100 & 0.76 & 0.80 \\
\hline & & & 120 & 0.72 & 0.83 \\
\hline & & & 200 & 0.72 & 0.85 \\
\hline \multirow{6}{*}{3.58} & \multirow{6}{*}{4.58} & \multirow{6}{*}{5.58} & 20 & 0.78 & 0.85 \\
\hline & & & 60 & 0.80 & 0.87 \\
\hline & & & 80 & 0.81 & 0.88 \\
\hline & & & 100 & 0.81 & 0.88 \\
\hline & & & 120 & 0.83 & 0.90 \\
\hline & & & 200 & 0.85 & 0.90 \\
\hline \multirow{6}{*}{4.58} & \multirow{6}{*}{5.58} & \multirow{6}{*}{6.58} & 20 & 0.80 & 0.90 \\
\hline & & & 60 & 0.88 & 0.92 \\
\hline & & & 80 & 0.88 & 0.89 \\
\hline & & & 100 & 0.89 & 0.96 \\
\hline & & & 120 & 0.89 & 0.96 \\
\hline & & & 200 & 0.89 & 0.97 \\
\hline
\end{tabular}

\section{Conclusion}

The present article deals with parameter estimation of generalized Paretodistribution under 3 step stress ALT plan. The objective is to plan a test that achieves the best reliability estimates. Two types of relationship are assumed between scale parameter and Stress. One links scale parameter linearly with stress while other have quadratic relationship. Comparison between both is shown by calculating estimates and their respective error. Efficiencies for both plans are calculated for different level of stress. Apart from that the results of optimal design of step-stress ALT for different sample size is shown. The performance of step-stress testing plans and model assumptions are generally evaluated by the properties of the maximum likelihood estimates of model parameters. Estimates of quadratic are more stable with relatively small Mean absolute error as sample size increases. Maximum likelihood estimators are consistent and asymptotically normally distributed. In short, it may be concluded that the present step stress ALT plan works well 
and has a good choice to be considered in the field of accelerated life testing.

\section{References}

[1] Alhadeed, A. A. and Yang, S. S. (2005). Optimal Simple StepStress Plan for Cumulative Exposure Model Using LogNormal Distribution. IEEE Transaction on Reliability, 54(1), 64-68.

[2] Bai, D. S., Kim, M.S. and Lee, S. H. (1989). Optimum Simple Step-Stress Accelerated Life Tests with Censoring. IEEE Transactions on Reliability, 38(5), 528-532.

[3] Bander-Al Zahrani (2012). Maximum Likelihood Estimation for Generalized Pareto Distribution under Progressive Censoring with Binomial Removals. Open Journal of Statistics, Scientific Research, 2, 420-423. http://dx.doi.org/10.4236/ojs.2012.24051

[4] Fard, N. and Li, C. (2009). Optimal Simple Step Stress Accelerated Life Test Design for Reliability Prediction, Journal of Statistical Planning and Inference, 139(5), 17991808.

[5] Hunt, S. and Xu, X. (2012). Optimum Design for Accelerated Life Testing with Simple Step- Stress Plans. International Journal of per formability Engineering, 8(5), 575- 579.
[6] Khamis, I. H. and Higgins J. J. (1996). Optimum 3-Step StepStress Tests. IEEE Transactions on Reliability, 45(2), 341-345.

[7] Khamis, I. H. (1997). Optimum M-Step, Step-Stress Design with K Stress Variables. Communicationsin Statistics, Simulation and Computation, 26(4), 1301-1313.

[8] Khamis I.H., and Higgins J.J. (1998)., A new model for stepstress testing, IEEE Transactions on Reliability, 47,131

[9] Lu, M.W. \& Rudy, R.J. (2002.) Step-stress accelerated test, International Journal of Materials \& Product Technology, 17 (5-6), 425-434.

[10] McSorley, E.O., Lu, J.C. \& Li, C.S. (2002). Performance of parameter-estimates in step stress accelerated life-tests with various sample-sizes, IEEE Transactions on Reliability, 51, (3), 271-277.

[11] Miller, R., and Nelson, W. B. (1983). Optimum Simple StepStress Plans for Accelerated Life Testing. IEEE Transactions on Reliability, R-32(1), 59-65

[12] Nelson, W. (1990), Accelerated Testing, Statistical Models, Test Plans and Data Analysis. John Wiley \& Sons.

[13] Xiong, C. (1999) Step stress model with threshold parameter, Journal of Statistical Computation and Simulation, 63, 349360 . 